\title{
Rehabilitation Center Planning using Multi-agent Systems
}

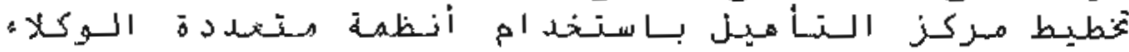

Sherif E. Hussein

Mansoura University, Faculty of Engineering, Computer \& Systems Department, Egypt.

$$
\text { ملخه البحت }
$$

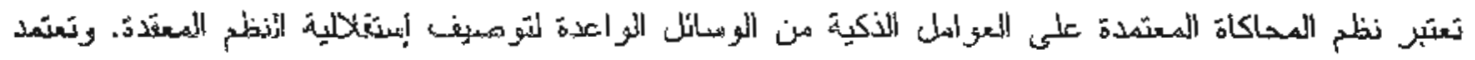

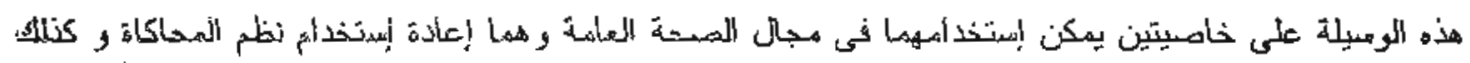

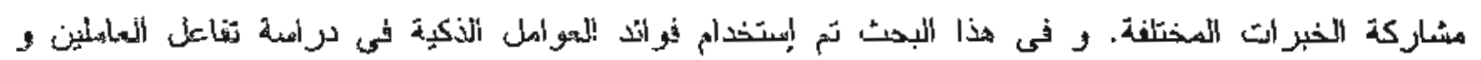

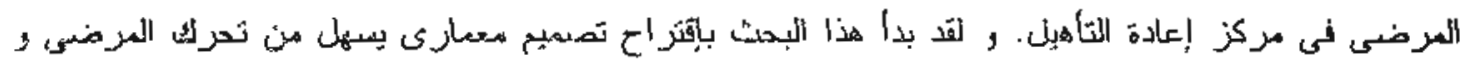

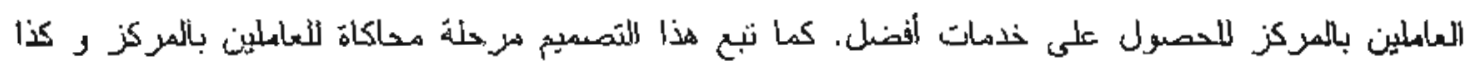

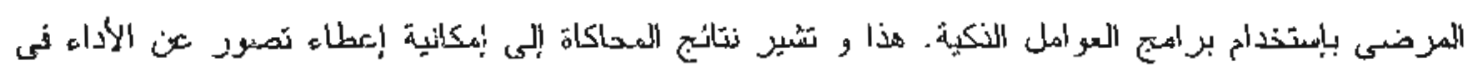

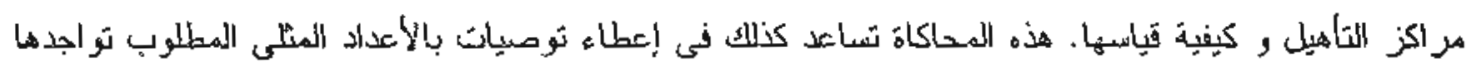

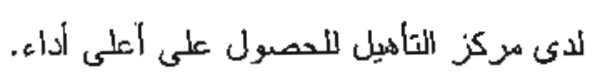

Abstract. Agent-based modeling and simulation is a promising technique utilized to study and simulate interdependencies of complex intrastruclures. This technique has two relevant properties which could be exploited in the field of healthcare, namely, the re-use of simulation models and the sharing of expertise. This research proposes a methodology to exploit the benefits of multi-agent systems with respect to the study of interdependencies between rehabilitation center personnel. The design procedure began by proposing an architectural layout which can best facilitate overall patient movement and improve healthcare services provided. Then, a simulation of the various personnel, along with the patients, was analyzed and implemented, using NetLogo, an agent-based simulation engine and environment. The simulation results showed how multi-agent systems could shed light on interdependent system performance and assist in their quantification. These results could then be used to make recommendations conceming the ideal number of personnel to be allocated to the rehabilitation center, thus ensuring its maximum performance.

\section{INTRODUCTION}

The planning of a rehabilitation center has a direct impact on the speed of recovery for patients with either traumatic brain or spinal cord injuries. Some of the reasons for this include the distance between one therapy area to the next and the lack of commurication between therapists and physiatrists. The overall nature of some facilities might be dark and depressing. The lack of these factors can definitely be a detriment to the patient's recovery. Successful recovery of a person can also be affected adversely by slack time. Quicker a person receives treatment, better are his chances for maximum benefit [1].

Due to long distances between treatment areas, some patients may receive more or less nursing observation than others. Therefore, the goal of planning the layout of the patient wings must be to minimize distances for the nurses. This is also beneficial in the need to provide 
minimize distances for the nurses. This is also beneficial in the need to provide the patient with economical, high-quality care [2]. Architect Lewis J. Sarvis investigated the amount of travel time nurses spend traveling between the centralized nurses' station and patient beds. He determined that nurses spent as much as $40 \%$ of their work day walking to and from the patients' beds [3]. Therefore, reducing the time nurses spend walking between these areas and the resulting increase in direct nursepatient contact has become a major factor in rehabilitation center planning.

In order to simulate the working environment in the rehabilitation center, different personnel interactions need to be decentralized and complex systems. Every person, either working or being treated in the center, needs to utilize his own memory and reactive behavior plus his ability to leam and plan. The simulation of such behavior can be modeled using multi-agent systems (MAS).

A MAS consists of a number of agents that are capable of being autonomous, proactive, adaptive and socially active, to communicate and interact sociability with each other. These agents may interact with each other both directly (through communication and negotiation) or indirectly (by acting on the environment). Agents may be able to cooperate for their mutual benefit or they may compete to serve their own interests. In a multi-agent system, an agent receives an indication of the current state of the environment as data input in each step of the interaction. The agent then performs an action which generates an output to change the state of the environment to a more desirable state by providing a reward or penalty. After the agent receives an offer from an opponent, it analyses the offer, modifies its solution and makes a conclusion. The updated conclusion then becomes the agent's a priori knowledge [4]

MAS have been used successfully in a few healthcare and planning applications. Xjao et al. [5] presented an adaptive security model for MAS and showed its potential application in a clinical trial to develop a prototype tumour classification system. Sud et al. [6] used a new approach for real-time multi-agent planming in pursuit-evasion, terrain exploration and crowd simulation scenarios which involved hundreds of moving agents, each with a distincl goal.

In this research the architectural analysis procedure was used to decide which design is superior taking the functionrelated space and ease of flow for the different personnel in the premises into consideration. After the design is an MAS stage which simulates the rehabilitation center environment, with different personnel modeled as agents, to evaluate a number of performance criteria that reflect the quality of the rehabilitation center and the strength of its work effectiveness.

\section{THE REHABILITATION CENTER LAYOUT}

Basically, a rehabilitation center must contain an area for the accommodation of patients which is easily accessible to the cafeteria, therapy area and visitation area. The environment in which the patient will be accommodated for the perjod of his stay must be cheerful and secure, with continuous supervision by various personnel. 


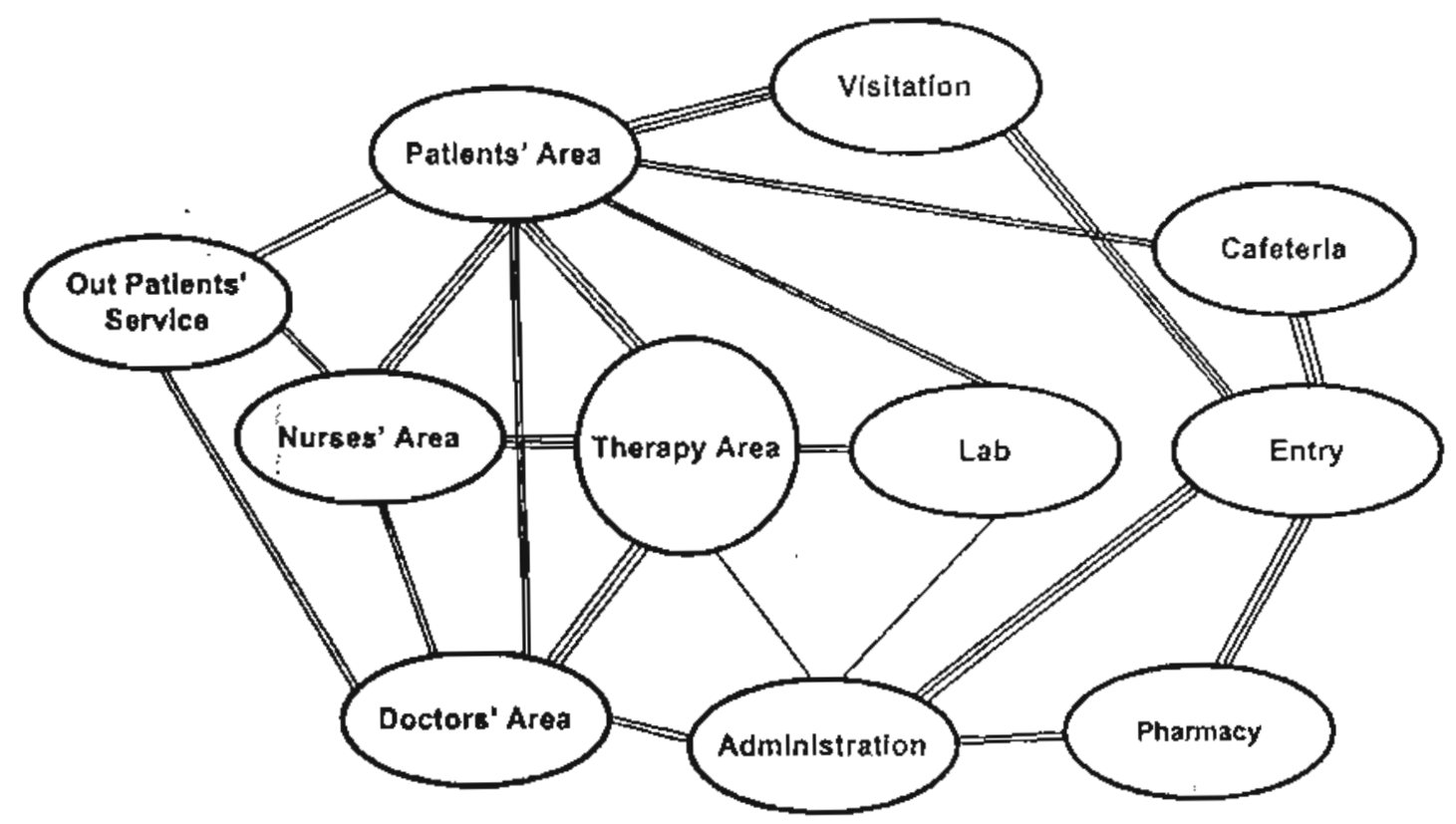

Fig. 1: A bubble diagram for the different functional areas of a rchabilitation center that refiect the strenglis of their relationships.

Other important areas might be the doctors', nurses', laboratory and plarmacy areas, plus the outpatient services, - entry and administration officcs. The nurses' area needs to be designed in such a way as to afford easy access to patients.

Main architectural designs may be radial, linear or clustered. Radial design provides a dominant central space from which a number of linear sections radiate outward. Linear design essentially employs a series of spaces which can be directly related to one: another or linked through another separate and distinct linear space. Lastly, clustered design makes use of repetitive, cellular spaces that have similar functions and share a common visual trait such as shape or orientation [7].

The proposed design in this research is a combination of both the radial and the clustered design, and aims to utilize the space in such a way as to offer the patient easy and convenient access.

\section{ARCITECTURAL DESIGN}

The first step in the architectural design was to detemine the various functional areas of the rehabilitation center and then to decide the importance of the relationships between each function as shown in figure 1.

Figure 1 shows the function-related areas that enable the architect to decide how close each area must be with respect to the other arcas. This diagram can be replicated many times using different arrangements, until an ideal arrangement is found. An additional complementary step is performed to check the traffic circulation and movement of the patients and other personnel who play a role in patient treatment, in order to decide which design will best facilitate smooth movement [8]. A floor plan design is 


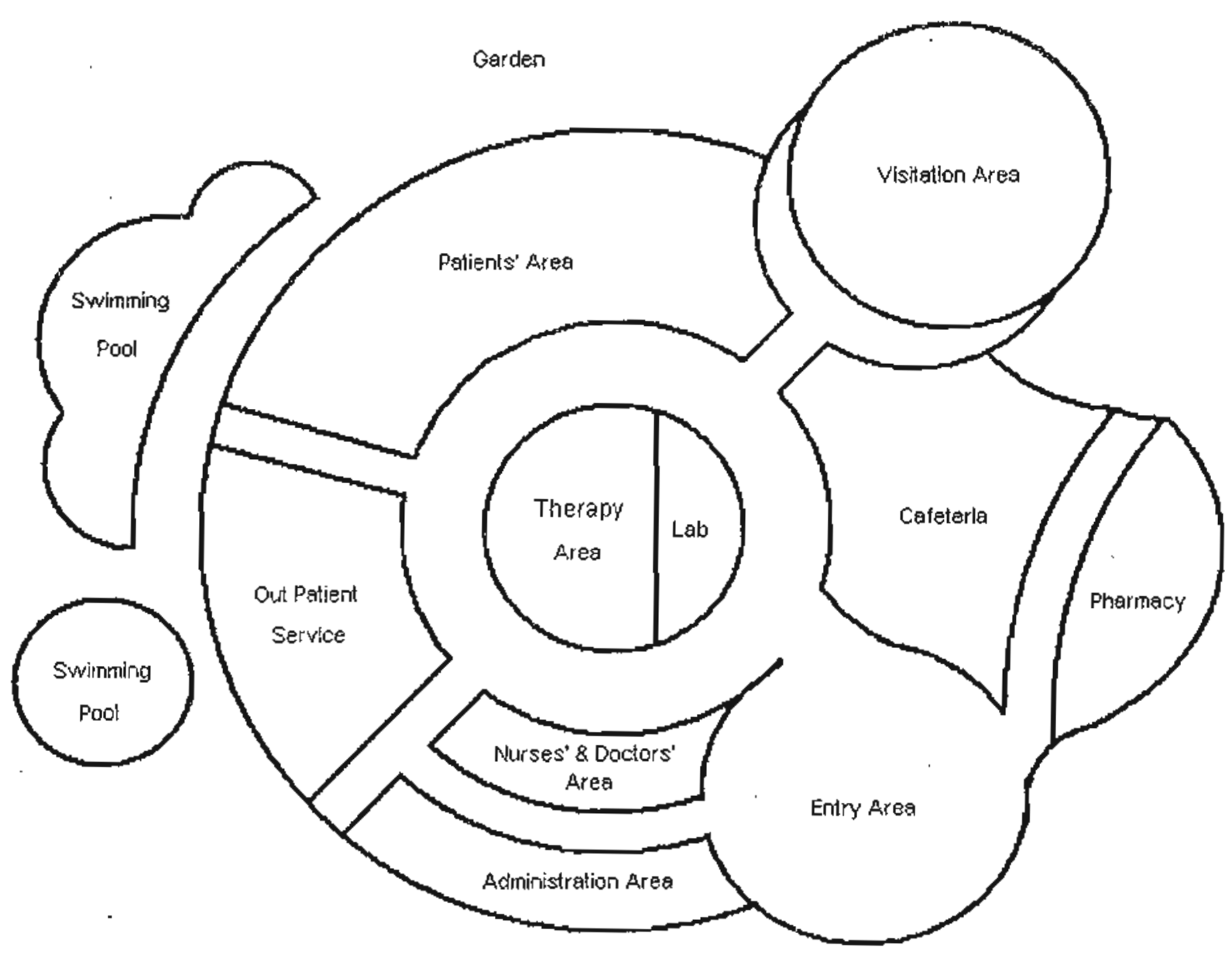

Fig. 2: A relabilitation center foor lyyouf illustrating the diflerem ancas of the promiscs

then developed, based on the two previous steps, in such a manner as to take into account all the constraints surrounding the system, as shown in figure 2 .

\section{SYSTEM MODELING}

Agent architecture involves the fundamental mechanisms underlying the autonomous components that support effective behavior in real-world, dynamic, open environments. These architectural components can be divided into four main groups: logic-based, reactive, belief-desire-intention (BDI), and layered architecture.
BDI architeciure is probably the most popular type for agents. It has its roots in philosophy and offers a logical theory which defines mental attitudes including belief, desire and intention, using modal logic. BDI architecture includes and uses an explicit representation for an agent's beliefs, desires, and intentions [9]. The advantage of this approach, as it is utilized for this research, is that the interpreter is able to stop the program at any time, save the state and execute a different plan or intention, if necessary. 


\subsection{Designing Agents}

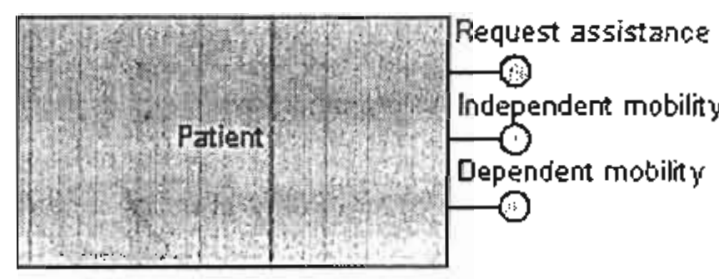

(a)

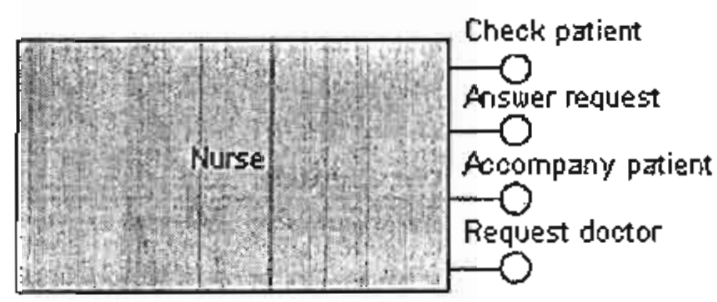

(b)

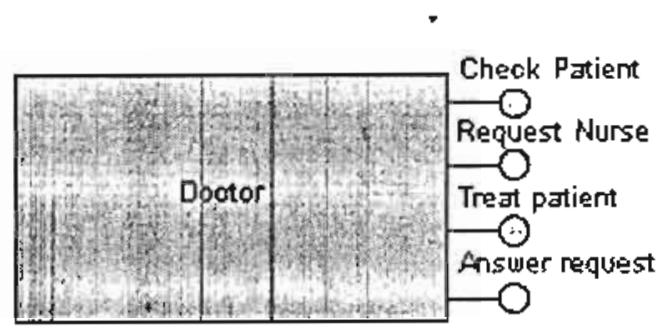

(c)

Fig. 3: Agent diagrams for (a) the patient, (b) the nurse, and (c) the doctor.

The design process begins by assigning the agent to one of three different groups that has been selected for the simulation process. These groups are patients, nurses and doctors. It is important to mention here that the doctors' group includes physiatrists, neuropsychologists, physical therapists, occupational therapists, speech therapists and recreational therapists. The grouping of different medical specialties into one unit serves to provide collaborative and interactive treatment. The next step is to define the different roles an agent will play, as shown in figure 3 . The roles defined for the patients' group are to request assistance from the nurse, engage in independent mobility or move with the help of a nurse. The defined roles for the nurses' group are to check on a patient, answer a patient request, request a doctor's attention or chose and accompany a patient. The roles for the doctors broup include checking paticnt, requesting a nurse, answering a nurse's request and treating a patient. These groups and their roles provide several pieces of information regarding cooperation, coordination and facilitation of information exchange [10].

\subsection{Protocols}

A set of rules represents the legal sequences of messiges between agents. This protocol is arranged around a frame that conlains lisc participants in an interaction, along with message sequences. Figure 4 slows two agents from the patients and the nurses groups. The patient requests the attention of a nurse and wails for the response. He calls the nurses, of which the total number is $\mathrm{m}$. lrom those, $\mathrm{n}$ of the nurses are busy while o of them are available. He chooses from the available o nurses closest to him. The nurse may arrive, or fail to arrive after a certain waiting time, set by the patient, has expired. 


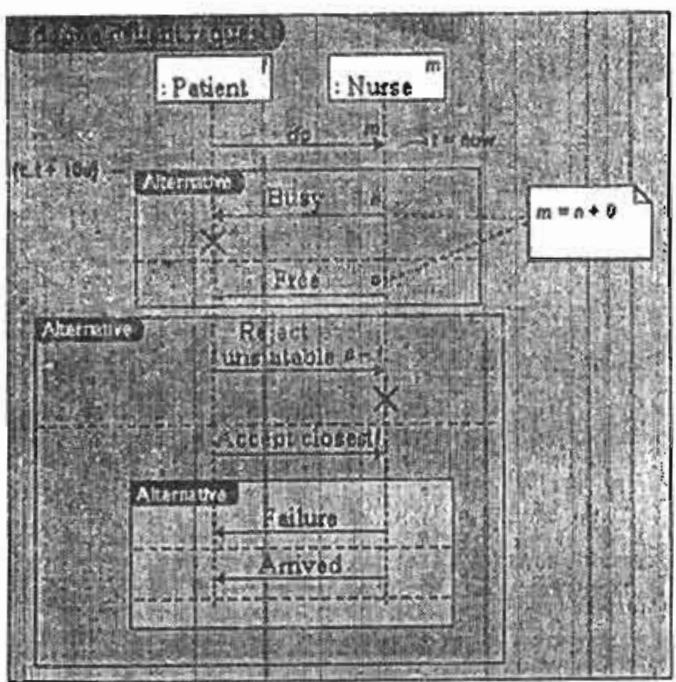

Fig. 4: Sequence diagram for a palient requesting assistancc from a nurse.

\subsection{Goals, Plans and Actions}

The modeling process's next step is to define and integrate goals, plans and actions within the agent shell. specifically, to associate actions with services, protocols and events. The fundamental unit of a goal is an action which represents a specific task the agent must perform. The agent itself initiates an action, such as requesting attention from a nurse, checking a patient or accompanying a patient to achieve an objective. Conditions can interfere with the execution of a specific action and can refer to a belief, desire or intention (BDI) modality. A precondition might be a call to a nurse by a patient or a call to a doctor by a nurse.

The goal of the patient agent is to calculate the average waiting time which in turn will reflect the patient's satisfaction with the response that he/she expertences; this is an indication of the quality of service offered by the center. On the other hand, the goal of the nurse and doctor agents is to calculate the average service, walking and idle times. It is important to note here that the higher the average service time, the better, but that doesn't necessary mean that the average waiting time for the patient will be minimized. On the contrary, the higher the average service time, busier and less responsive to calls the nurses and doctors become. The simulation reflects these factors for different numbers of patient, nurse and doctor ratios. An increase in the number of nurses and doctors means a decrease in both the average waiting time for patients and the average service time by the nurses and doctors. Therefore, a careful selection needs to be considered in a real environment where performance may not be as good as expected and the question will turn to be how much less compared to the simulation environment as criteria to evaluate the personnel effort working in the premises.

The patient agent is able to check the distances each free nurse walks to reach him. This behavior helps in determining the closest one and consequently, minimizing the average waiting time.

The nurse and doctor agent can be wandering around on a predetermined path or may be found in their designated area when they are not treating a patient. These activities are governed by probabilistic behavior and can be set during the simulation. A doctor's activity can initiate the selection of a patient to be checked or call for his attendance in the therapy area to administer treatment to a specific patient. This activity triggers the action of a nurse to accompany the patient if he needs assistance or the patient may make his own way, if he is capable of independent mobility. As 

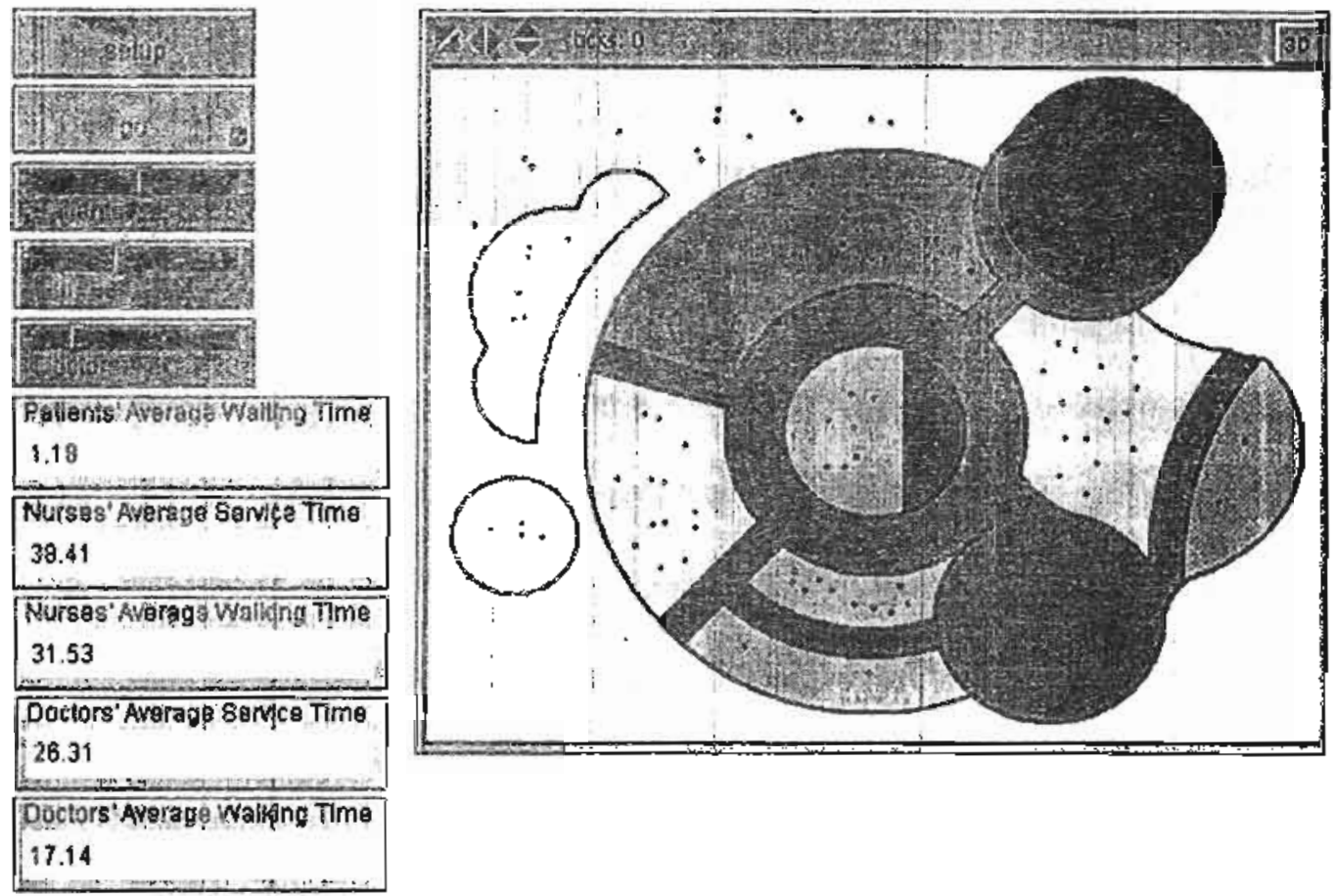

Fig 5: A GUI for the system simulation will the ted circles representing paticnts, biuc eircles representing nurses, and yellow circles representing doctors.

treatment in the therapy area requires nurses, it is normal for the joctor to request a certain number of nurses to be present, regardless of the patient's mobility.

\section{SYSTEM SIMULATION}

A complete agent-based system requires an infrastructure to facilitate message transport, direciory services, notifications of events and delivery services. For this research, NetLogo has been used for simulation of the agents. Netlogo is a programmable modeling environment, which can be used for simulating certain natural and social phenomena and is particularly well suited for modeling complex systems developing over time. The modelers can specify instruccions to hundreds or even thousands of independent agents which can all operate in parallel. This makes it possible to explore the connection between the micro-level behavior of individuals and the macro-level patterns that emerge from the interaction of many individuals. NetLogo is the next generation in a series of modeling languages with agents that began with StarLogo [11]. It is a medium written entirely in Java; therefore it can be installed and activated on most important platforms.

The simulation uses three kinds of agents: patients, nurses and doctors. They move and communicate in an environment that represents a rehabilitation center layout. The layout has been modeled utilizing different colors to guide the agent movements. A patient agent may know his health condition and can move around a few predetermined areas. The patient agent is programmed with a parameter that stores 
information about the average time that elapses between the patient's request for a nurse and the time he/she actually arrives. Nurse and doctor agents also have a few parameters that represent the effort they expend while doing their work. One of these variables stores the total time spent in walking, compared to the total working time. The other variable represents the total time spent in treating or attending a patient, compared to the total working time. These parameters aid in quantifying the performance of the center for a determined number of patient, nurse and doctor ratios. They can greatly assist in deciding on the total number of staff that may be needed so as to offer satisfactory services.

Figure 5 shows the different variables that can be changed during the simulation process. The variables include the number of patients, nurses and doctors that play important roles in the simulation process. The ratio of the number of nurses and doctors to the number of patients directly affects the average waiting time, service time and walking time; these are expressed as a percentage of the total working time during the period that the patients are being cared for in the rehabilitation center. This, in turn, ajds in choosing the optimal number of personnel for the premises.

\section{CONCLUSION}

The simulation presented in this research not only presents appropriate architecture for a rehabilitation center that would offer a suitable environment for patient treatment, but also provides a basis which a manager can rely upon when simulating the best environment for a rehabilitation center. This environment can help the manager in deciding the appropriate number of staff that will be needed to provide satisfactory treatment and provides a baseline for the comparison of the activities that each worker is expected to perform.

As one would expect from a multi-agent system, this model simulation can be used in the study of an agent's behavior in various situations. Furthermore, it can be used to identify possible enhancements which may be instrumental in improving the performance of a rehabilitation center. This model can also be used in creating educational software to be utilized when considering different scenarios which the management and the staff may be faced with during the course of their work.

\section{REFERENCES}

1-E. C. Brawley, Strategies for Creating Better Care Environments, Designing for Alzheimer's disease, New York, John Wiley \& Sons Inc., 1997

2- F. C. Salmon and C. F. Salmon, Rehabiliation Center Planning, University Park, Pennsyivania, The Pennsylvania State University Press, 1959.

3- S. A. Kliment, Building Type Basics for Health-Care Facilities, New York, John Wiley \& Sons, 2000.

4- M. Wooldridge, Reasoning about Rational Agents, MIT Press, 2000.

5- L. Xiao, A. Peet, P. Lewis, S. Dashmapatra, C. Saez, M. Croitoru, J. Vicente, H. Gonzalez-Velez, M. L. Ariet, "An Adaptive Security Model for Multi-agent Systems and Application to a Clinical Trials Environment," 31 st Annual 
International Computer Software and Applications Conference, pp. $261-268$ , July 2007.

6- A. Sud, E. Andersen, S. Curtis, M. C. Lin , D. Manocha, "Real-Time Path Planning in Dynamic Virtual Environnents Using Multiagent Navigation Graphs," IEEE Transactions on Visualizalion and Computer Graphics. Vol. 14, No. 3, pp. 526-538, May/June 2008.

7- F. D. K. Ching, Form, Space, and Order, Wiley, 2nd edition, 1996.

8- D. E. Brown, M. Fox, M. R. Pelletier and L. Hoffman, Sustainable Architecture White Papers, New York, Earth Pledge Foundation, 2001.

9- F. L. Bellifemine, G. Caire and D. Greenwood, Developing Multi-Agent Systems with JADE, Wiley, 2007.

10- M.-P. Huget, "Agent UML notation for multiagent system design," Internet Computing, IEEE, Vol. 8, No. 4, pp. 63-71, July-Aug. 2004.

11- U. Wilensky. NetLogo. http//ccl.northwestern.edu/netlogo/. Center for Connected Learning and Computer-Based Modeling, Northwestern University. Evanston, IL., 1999.

12- J. M. Epstein, Generative Social Science: Studies in Agent-Based Computational Modeling, Princeton University Press, 2007.

13- M. d'Inverno, Understanding Agent Systems, Springer, 2003.

14- L. Otsuka, H. V. da Rocha, D. M. Beder, "A Multi-Agent Formative Assessment Support Model for Learning Mapagement Systems," IEEE Intemational Conference on Advanced Learning Technologies (ICALT 2007), pp. 85-89, July 2007

15- L. Bullivant, 4dspace: Interactive Architecture, Academy Press, 2005.
16- Tony Bertauski, Plan Graphics for the Landscape Designer, Prentice Hall, 2nd edition, 2006.

17- M. Hoogendoom and J. Treur, "An Adaptive Multi-agent Organization Model Based on Dynamic Role Allocation," 2006 IEEE/WIC/ACM International Conference on Intelligent Agent Technology (IAT'06). pp. 474-481, Dec. 2006.

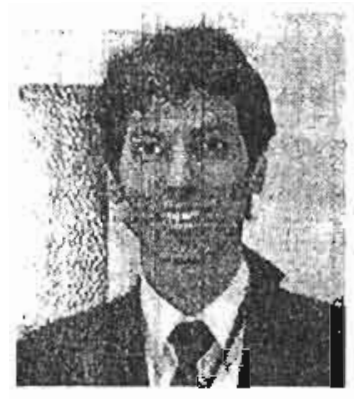

Sherif E. Hussein received the BSc and $\mathrm{MSc}$ in Computer Science from Mansoura University, Mansoura, Egypt with honor in 1994 and 1997 respectively and the $\mathrm{Pl} \mathrm{h}$ in Biomedical Engineering from University of Strathclyde, Glasgow. UK in 2003 and has been awarded the ORS and the Strathclyde studentship during his PhD. $\mathrm{He}$ is a compuler technical consultant for the Egyptian ninistry of Higher Education and an Assistant Professor in Computer and Systems Department, Mansoura University, Egypt. His research interests include Biomechanics, medical image and signal processing, biological modeling and artificial intelligence. Sherif is a member of the Institute of Electrical and Electronics Engineers IEEE, the International Federation for Artificial Organs IFAO, the Institute of Engineering and Technology IET, and a council member in the International Committee on Measurements and Instrumentation ICMI. 